\title{
Annual and semiannual variations of vertical total electron content during high solar activity based on GPS observations
}

\author{
M. P. Natali and A. Meza \\ Facultad de Ciencias Astronómicas y Geofísicas, Universidad Nacional de La Plata Paseo del Bosque s/n, (1900), La Plata, \\ Argentina \\ Consejo Nacional de Investigaciones Científicas y Técnicas (CONICET), Av. Rivadavia 1917, Buenos Aires, Argentina
}

Received: 22 September 2010 - Revised: 22 March 2011 - Accepted: 28 March 2011 - Published: 18 May 2011

\begin{abstract}
Annual, semiannual and seasonal variations of the Vertical Total Electron Content (VTEC) have been investigated during high solar activity in 2000. In this work we use Global IGS VTEC maps and Principal Component Analysis to study spatial and temporal ionospheric variability. The behavior of VTEC variations at two-hour periods, at noon and at night is analyzed. Particular characteristics associated with each period and the geomagnetic regions are highlighted.

The variations at night are smaller than those obtained at noon. At noon it is possible to see patterns of the seasonal variation at high latitude, and patterns of the semiannual anomaly at low latitudes with a slow decrease towards mid latitudes. At night there is no evidence of seasonal or annual anomaly for any region, but it was possible to see the semiannual anomaly at low latitudes with a sudden decrease towards mid latitudes. In general, the semiannual behavior shows March-April equinox at least $40 \%$ higher than September one. Similarities and differences are analyzed also with regard to the same analysis done for a period of low solar activity.
\end{abstract}

Keywords. Ionosphere (Ionospheric irregularities)

\section{Introduction}

It is well known that the variations of the Earth's ionosphere are complicated. Historically, ionospheric observations were compared with the Chapman (1931) theory, based on that the electron concentrations should vary regularly with the solar zenith angles. Major deviations from this theory are named "anomalies". In fact, the F2 layer is anomalous in many ways. Several authors have analyzed the annual, seasonal and semiannual anomalies of the ionosphere by using

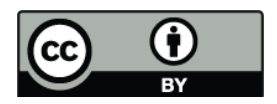

Correspondence to: M. P. Natali (paula@fcaglp.unlp.edu.ar)
F2-layer peak electron content $(\mathrm{Nm} \mathrm{F} 2)$ and TEC (Rishbeth, 1998; Rishbeth et al., 2000; Zou et al., 2000; Liu et al., 2009; Zhao et al., 2005; Meza and Natali, 2008). These anomalies can be summarized as follows:

Winter or seasonal anomaly: noon values of $N m \mathrm{~F} 2$ are greater in winter than in summer, whereas the Chapman theory leads us to expect the opposite. The anomaly disappears at night. Rishbeth and Setty (1961) proposed that the seasonal variation of $N m \mathrm{~F} 2$ is related with change of composition and Johnson (1964) suggested that this change was a result of summer hemisphere being heated and the lighter neutral constituents being convected to the winter hemisphere. So, Torr and Torr (1973) explained this variation in terms of the winter/summer thermospheric composition ([O]/[N2]) change including vibrationally excited nitrogen. The winter anomaly falls off in amplitude and area with decreasing solar activity as would be expected to happen with decreasing energy input and consequent reduction in the convection activation mechanism. The daytime seasonal anomaly is strong in the bottomside ionosphere and extends up to about $400 \mathrm{~km}$ altitude. At night the anomaly disappears. This is due to the effect of the strong nighttime equatorward wind in summer and the values of Ne in summer far exceed those in winter, especially at altitudes above $200 \mathrm{~km}$. Zhao et al. (2007) showed that the seasonal anomaly is more significant near the pole region.

Semiannual anomaly: $N m \mathrm{~F} 2$ is abnormally large at equinoxes. The explanation of the semiannual anomaly is more difficult. Several authors have suggested a number of mechanisms to explain the observed variation: Yonezawa (1971) proposed that the semiannual variation of $N m \mathrm{~F} 2$ is related with the variation of the upper atmosphere temperature, Torr and Torr (1973) suggested that this is due to semiannual variation in neutral densities associated with geomagnetic and auroral activity. Mayr and Mahajan (1971) showed that the semiannual effect appears as a persistent feature of the ionosphere which, demonstrably, is not related to

Published by Copernicus Publications on behalf of the European Geosciences Union. 
fluctuations in the $10.7 \mathrm{~cm}$ noise or the EUV radiation; this gives support to theories that attribute the semiannual effect to variations in the lower atmosphere. Although theoretically predicted temperature variations (Volland, 1969) could quantitatively account for the observed semiannual variations in the height of the F2 peak, the variations in $N m \mathrm{~F} 2$ require additionally significant variations in the neutral composition at lower heights and evidence for this was found in rocket-borne $[\mathrm{O}] /\left[\mathrm{O}_{2}\right]$ measurements at $120 \mathrm{~km}$. Millward et al. (1996) using the Coupled Thermosphere-IonospherePlasmasphere model (CTIP) found the semiannual variation in $N m \mathrm{~F} 2$ noontime, a common feature of the F2-layer, particularly at low latitudes and in the Southern Hemisphere at mid-latitudes. Results from the model reveal such variation, most prominently, at mid-latitudes, in the South American sector and concluded that this phenomenon is intimately related to the large offset of the geomagnetic axis from Earths spin axis in the Southern Hemisphere. Chaman Lal (1995) constructed a planetary index of the critical frequency of F2 layer, it showed marked semiannual maxima around the equinoxes in the similar way that Dst geomagnetic index (which measures the intensity of the ring current), so he discussed the relation of the solar wind energy on these processes (Chaman Lal, 2000). Ma et al. (2003) suggested that the semiannual variation of the diurnal tide in the lower thermosphere induces the semiannual variation of the amplitude of the equatorial electrojet, this causes the variation of amplitude of ionospheric equatorial anomaly through fountain effect and this process induces the semiannual variation of the low latitude $N m \mathrm{~F} 2$. The semiannual variation appears around the globe at daytime, and except in low latitude region and in South American sector ( $\mathrm{Li}$ and $\mathrm{Yu}, 2003$ ) there is no obvious semiannual variation of $N m \mathrm{~F} 2$ in the nighttime. The amplitude of this variation has close relationship with the solar activity, they have asymmetrical structures between the two hemispheres (larger at the Northern Hemisphere) and they have longitude difference (Ma et al., 2003). Balan et al. (2000) found a strong equinoctial asymmetry that exists near the ionospheric peak and above at all local times with the values of $\mathrm{Ne}$ in March equinox exceeding that September equinox by up $50 \%$.

Annual anomaly: in the world as a whole December $N m \mathrm{~F} 2$ is on average greater than June $N m \mathrm{~F} 2$, both by day and by night. A possible cause of the annual anomaly is the changing distance between Sun and Earth, but it is insufficient to account for the reporter annual anomaly, so other explanations were suggested. Yonezawa (1959) found that the average of observations of $N m \mathrm{~F} 2$ made at conjugate stations at December solstice exceeds that at the June solstice by around $20 \%$. Later, Yonezawa (1971) completed his work and found that the relative amplitude of the annual component in $N m F 2$ decreases slowly with increasing latitude and solar activity during daytime and is smaller at night compared to daytime. Buonsanto (1986) suggested that, at perihelion the greater flux of solar ultraviolet causes greater dis- sociation of molecular oxygen than aphelion but this hypothesis doesn't explain the totality of the F2-layer asymmetry. Su et al. (1998) proposed the dynamical processes cause the annual asymmetry; he analyzed the topside ionosphere at low latitudes. The observation showed a strong annual anomaly at all longitudes concluding that the differences between the solstice values of neutral wind (from coupling of the neutral gas and plasma) may make a significant contribution to daytime and the $\boldsymbol{E} \times \boldsymbol{B}$ drift velocity may slightly weaken the annual anomaly during daytime and strengthen the anomaly during the post-sunset period. Rishbeth and Müller-Wodarg (2006) concluded that dynamical influences of the lower atmosphere (below about $30 \mathrm{~km}$ ) are the most likely cause of the asymmetry.

Torr and Torr (1973) analyzed $N m \mathrm{~F} 2$ variations in winter maximum (seasonal), equinoctial maxima (semi-annual) and a component which peaks in December-January (annual). They constructed global maps that represent the different components at different solar activity. The solar-cycle changes in the domains of the annual and semiannual effects have yet to be explained, but may be connected with changes in the strengths of the global circulation. At low solar activity, there are regions of summer maximum (i.e. no anomaly) at equatorial and southern latitudes.

In recent years, a database of ionospheric VTEC derived from GPS observations produced by a worldwide network of continuously tracking stations, has been used to investigate the local and regional characteristics of various anomalies (Huang and Cheng, 1996; Wu et al., 2004; Liu et al., 2009; Astafyeva et al., 2008). The worldwide GPS network above mentioned is managed by the International GNSS (Global Navigation Satellite System) Service, known as IGS.

In May 1998, IGS created the Ionosphere Working Group (Feltens and Schaer, 1998). Among the scientific groups and institutes that are presently dedicated to ionospheric studies using GPS observations three of them can be distinguished as the most important: the Center for Orbit Determination in Europe (CODE), that belongs to the Astronomical Institute of the University of Bern, Switzerland (http://www.aiub.unibe.ch/igs.html); the NASA Jet Propulsion Laboratory (JPL) (http://iono.jpl.nasa.gov/), Pasadena, USA; and the Astronomy and GEomatics group (gAGE) (http://gage1.upc.es/), Barcelona, Spain. The Global Ionosphere Maps (GIMs) computed by each group is made available for the users as a file in the IONosphere map EXchange (IONEX) format (Schaer et al., 1998). The file contains all the information relative to the computation process and the VTEC information are presented in the form of a grid of 2.5 degrees in latitude and 5 degrees in longitude.

The numerical technique to be use in this paper to analyze the variability of the ionosphere is the Principal Component Analysis.

The Principal Component Analysis (PCA) method is regularly used by meteorologists and oceanographers as a tool for the analysis of the spatial or temporal variability of physical 
fields. PCA is the decomposition of a data set into a base of orthonormal functions which are directly determined by the data set itself. This mathematical procedure transforms a set of correlated variables into a number of uncorrelated variables called principal components. Several investigators have used the PCA technique to help isolating the cause of the sea-level change signals (Nerem et al., 1994, 1997). It has also been used for empirical ionospheric modelling (Zhao et al., 2005; Meza and Natali, 2008).

In this paper, the annual, semiannual and seasonal variations of the ionosphere during high solar activity throughout year 2000 are analyzed using Global IGS VTEC maps.

Applying the PCA technique on a time series of IGS VTEC global maps give us an efficient method to analyze the main ionospheric anomalies on a global scale. The behavior of VTEC variations at two-hour periods centered at 12:00 p.m. and 10:00 p.m. local time are analyzed.

After a brief description of the data acquisition and PCA method, this technique is applied to the data set. Then, the analysis of the results in the physical frame is described. Finally, the variabilities of the ionospheric anomaly trough IGS VTEC global maps are evaluated and discussed.

\section{Methodology}

\subsection{Principal Component Analysis}

PCA is well suited for the analysis of multi-variate time series. One main reason for that is that the technique can be used to identify spatial structures that have dominant contribution to the total variability together with their time evolution without the need to propose any particular a priori functional model.

PCA is used to express a correlated data set on a new orthonormal base of minimum dimension. The shape of the base functions is determined from the data set itself. This method is of special interest when the phenomena under study are not necessarily a superposition of well known simple components that would point other techniques e.g. Fourier analysis as more adequate.

In the next paragraph a brief description of the algebraic essentials of PCA is presented. For further details about algebraic foundations of PCA, see Preisendorfer (1988). A description of the technique with focus on its application for TEC analysis can be found on Meza and Natali (2008), Natali and Meza (2010).

Let $z(t, x)$ be VTEC measures, at point $x$ (latitude and longitude) in the atmosphere at time $t$. Let these measurements be taken over the set of locations $x=1, \ldots ., p$ at times $t=1$, $\ldots . . ., n$. The first step in PCA is to center the time series on their time averages. This is,

$z^{\prime}(t, x)=z(t, x)-\bar{z}(t, x)$ with,

$\bar{z}(t, x)=\frac{\sum_{t=1}^{n} z(t, x)}{n}$

These collections can be thought as $p \times 1$ (i.e., column) vectors $z^{\prime}(t)=\left\{z^{\prime}(t, 1), \ldots . ., z^{\prime}(t, p)\right\}$ forming a swarm of points about the origin of a p-dimensional Euclidean space $\left(E_{p}\right)$. Now it is possible to construct the symmetric scatter matrix, $\mathbf{S}$, in $E_{p}$.

$\mathbf{S}=\sum_{t=1}^{n} z^{\prime}(t) z^{\prime T}(t)$

This matrix has a set of $p$ orthonormal eigenvectors $\boldsymbol{e}_{j}=$ $\left[\boldsymbol{e}_{j}(1), \ldots \ldots, \boldsymbol{e}_{j}(p)\right]^{T}, j=1, \ldots, p$. These are the "empirical orthogonal functions (EOF)": empirical because they arise from data, orthogonal because they are uncorrelated over space:

$\sum_{x=1}^{p} \boldsymbol{e}_{j}^{T}(x) \boldsymbol{e}_{k}(x)=\begin{aligned} & 0 \ldots i f \ldots j \neq k \\ & 1 \ldots i f \ldots j=k\end{aligned} \quad j, k=1, \ldots ., p$

From these $\boldsymbol{e}_{j}$ we can construct the principal components (or amplitudes) of the data set, $\boldsymbol{a}_{j}(t)$ :

$\boldsymbol{a}_{j}(t)=\sum_{x=1}^{p} z^{\prime}(t, x) \boldsymbol{e}_{j}(x)=z^{\prime}(t)^{T} \boldsymbol{e}_{j} \quad\left(\right.$ analysis of $\left.z^{\prime}\right)$

$t=1, \ldots, n \ldots ; j=1, \ldots, p$

These $\boldsymbol{a}_{j}(t)$, can be thought of as a family of time series $\left\{\boldsymbol{a}_{j}(t): t=1, \ldots, n\right\}$. The most important property of these time series is that they are mutually uncorrelated, carrying information about the variance of the data set along the directions $\boldsymbol{e}_{j}$ :

$\sum_{t=1}^{n} \boldsymbol{a}_{j}^{T}(t) \boldsymbol{a}_{k}(t)=\begin{aligned} & 0 \ldots i f \ldots j \neq k \\ & l_{j} \ldots i f \ldots j=k\end{aligned} \quad j, k=1, \ldots ., p$

where $l_{j}$ is the $j$-th eigenvalue of $\mathbf{S}$. Finally, and most importantly, the original centered data set can be exactly represented in the form

$\boldsymbol{z}^{\prime T}(t, x)=\sum_{j=1}^{p} \boldsymbol{a}_{j}(t) \boldsymbol{e}_{j}^{T}(x) \quad\left(\right.$ synthesis of $\left.\boldsymbol{z}^{\prime}\right)$

$t=1, \ldots, n ; \ldots x=1, \ldots, p$.

By eigenvalue decomposition of the covariance matrix of VTEC variations $\left(z^{\prime}(t, x)\right)$ the PCA technique identifies those spatial structures of the ionosphere variability that have dominant contribution to the total variance (given by the sum of all eigenvalues). The spatial structure of the ionosphere variability is represented by the eigenvector $\left(\boldsymbol{e}_{j}(x)\right)$ and its temporal evolution is described by a series of coefficients $\left(\boldsymbol{a}_{j}(t)\right)$, called principal components. Eigenvector and principal components together are called mode. Modes are ordered according to decreasing eigenvalues, such that the first 

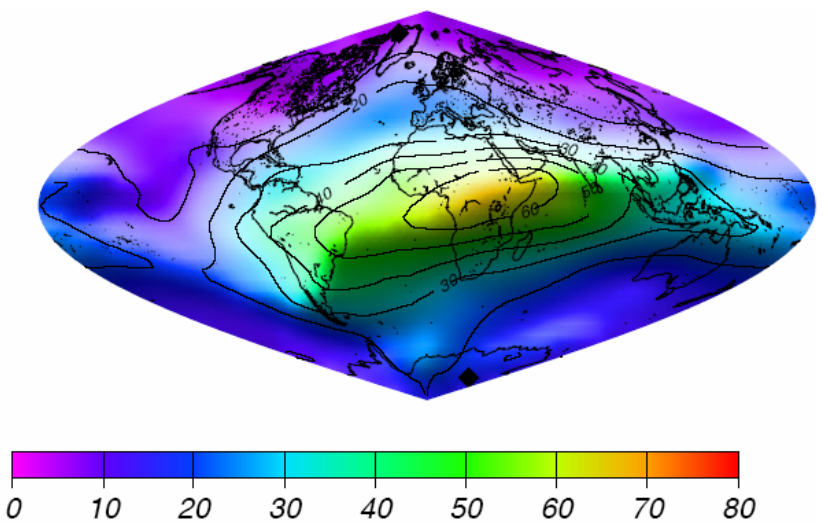

Fig. 1. IGS VTEC global map for the day 1 at noon.
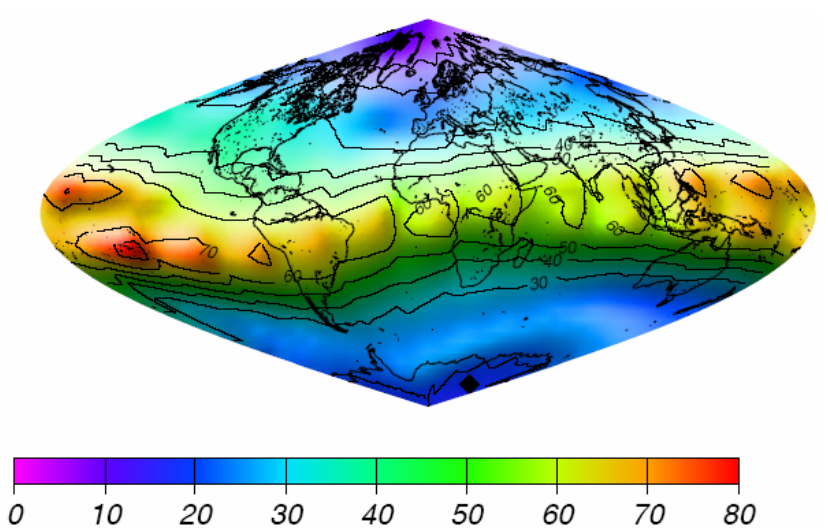

Fig. 2. IGS VTEC MAP after the reorganization of the data. This is a snapshot of the ionosphere for day 1 of 2000 at noon.

mode represents the largest part of the variance, the next mode the second largest part, etc.

The PCA analysis software we wrote for this work was based on the subroutines "jabob" and "eigsrt" from Numerical Recipes (1995). In many cases the scatter matrix $\mathbf{S}$ may become very large, so it is difficult to compute eigenvalues, so an alternative is to use Singular Value Decomposition over $z^{\prime}$.

\subsection{Chosen scenario}

The IONEX format allows the storage of snapshots of the electron density (including associated rms information) referring to particular epochs and to a 2-or even 3-dimensional, Earth-fixed grid. IONEX data supply a good estimation of the worldwide VTEC. These data provide VTEC values around the world at intervals of 2.5 degrees in latitude and 5 degrees in longitude (Fig. 1).

Global IGS VTEC maps during high solar activity (from January to December 2000) are used in this work. This VTEC maps show a global snapshot of the ionosphere every two hours. Therefore, the main geographical VTEC varia-
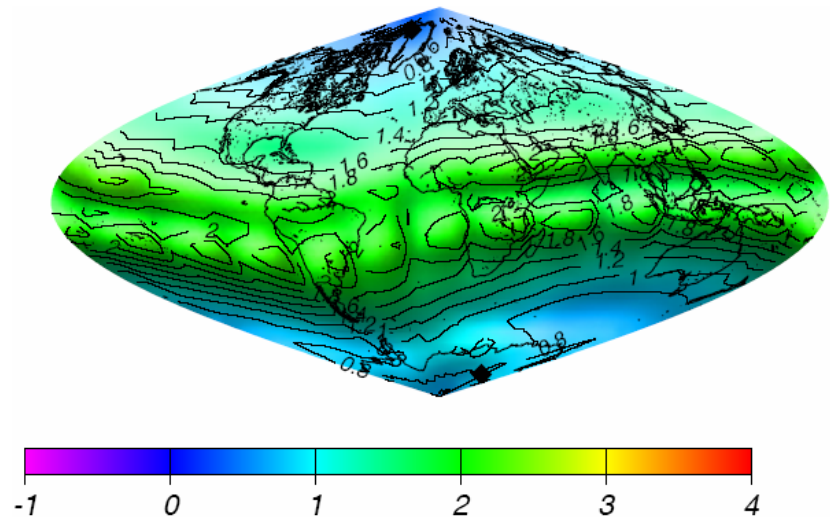

Fig. 3a. Spatial variation in the first mode at noon.

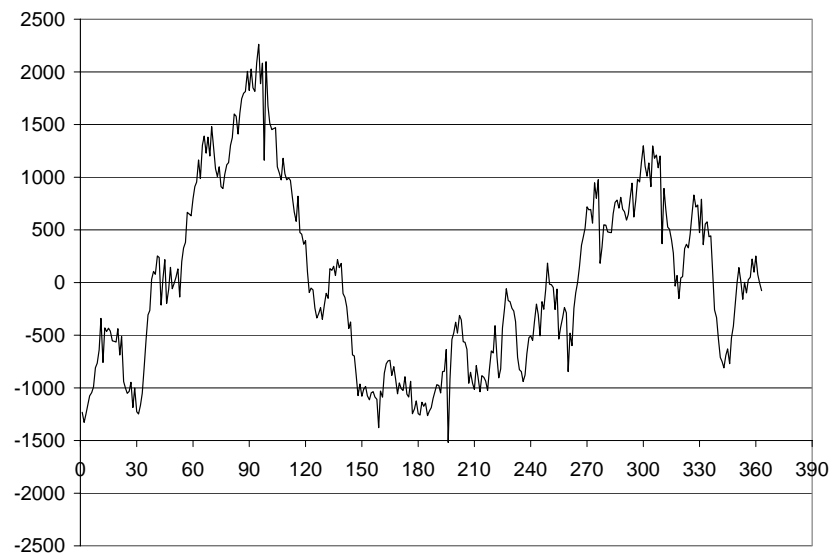

Fig. 3b. Time variation in the first mode at noon. The $x$-axis is the day of year (DOY) and the y-axis is the amplitude multiplied by a factor of $10^{-2}$.

tion that can be seen on them is the ionization due to solar radiation. Since we are not interested in analyzing that effect but the ionospheric response to similar conditions on different locations, we reorganized the VTEC data as follows: two different solar radiation conditions were selected for analysis: from each daily global data sets, composed by twelve VTEC maps, two maps were constructed. One of them corresponds to 12:00 p.m. local time worldwide; the other one corresponded to 10:00 p.m. The temporal series were constructed in the following way: Assuming that the ionosphere doesn't change in a two hour window, we took 30 degree slices from all VTEC maps for each day, centered on the same local time. These slices were then merged into a new VTEC map according to their central longitude. This procedure results in two new VTEC maps per day, corresponding to 12:00 p.m. and 10:00 p.m. worldwide. The complete data set consists on two series of VTEC maps covering year 2000 for 12:00 p.m. and 10:00 p.m., respectively. It is important to stress that there are no further processes applied to the data besides this sorting. No smoothing was applied in the process 

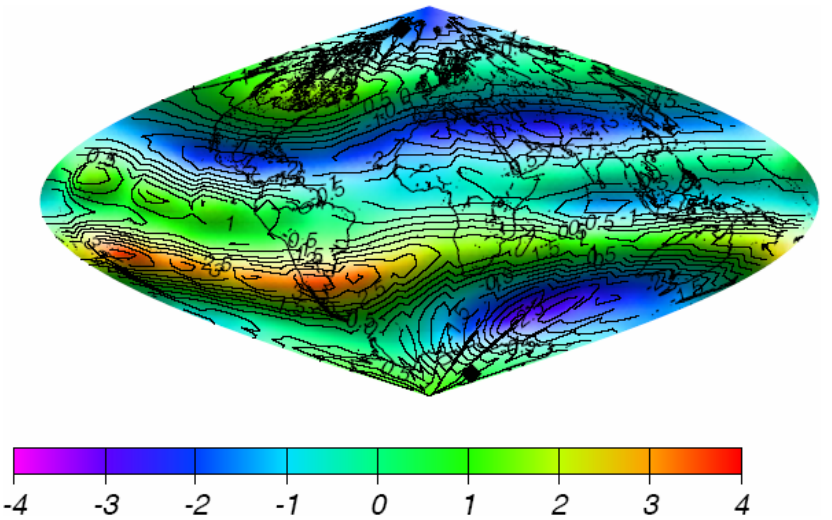

Fig. 4a. Spatial variation in the second mode at noon.

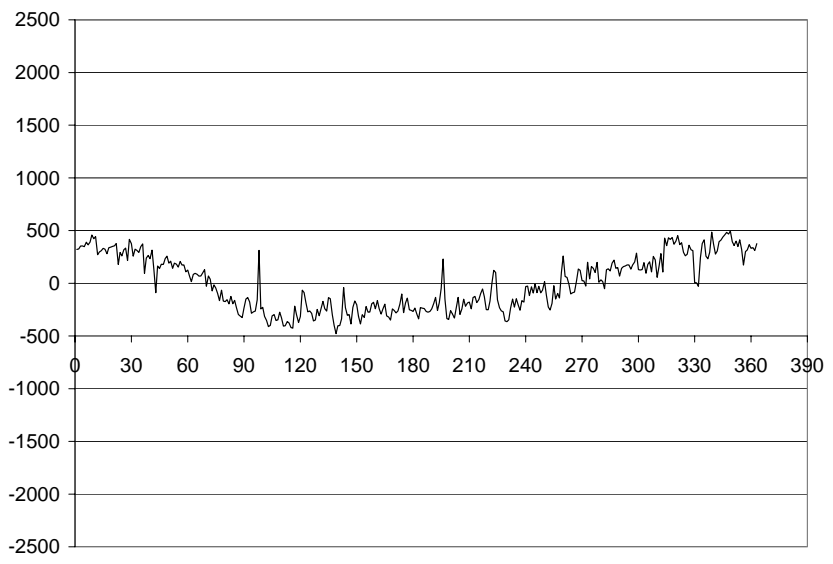

Fig. 4b. Time variation in the second mode at noon. The $\mathrm{x}$-axis is the day of year (DOY) and the y-axis is the amplitude multiplied by a factor of $10^{-}$.

of building these map time series (Natali and Meza, 2010). An example of the resulting daily maps for 12:00 p.m. for the day 1 is shown in Fig. 2.

\section{Results and discussion}

The VTEC behavior is analyzed during high solar activity for local time noon and night. These epochs correspond to two different time series once the VTEC grids are built for every day in year 2000. PCA analysis was applied to each time series in order to estimate the amplitudes $\left(a_{j}\right)$ and eigenvectors $\left(\boldsymbol{e}_{j}\right)$ associated to each equation data set (7).

For this dataset, we observed that the first two PCA modes already contain $80 \%$ of the total VTEC variability. Thus, in the following we analyze just these two modes for both epochs selected.

Modes 1 and 2 can be written as the products $a_{1}(t) e_{1}^{T}(x)$ and $a_{2}(t) e_{2}^{T}(x)$, respectively, where the $e_{j}$ contain the spatial variation and the $a_{j}$ contain the temporal variation of
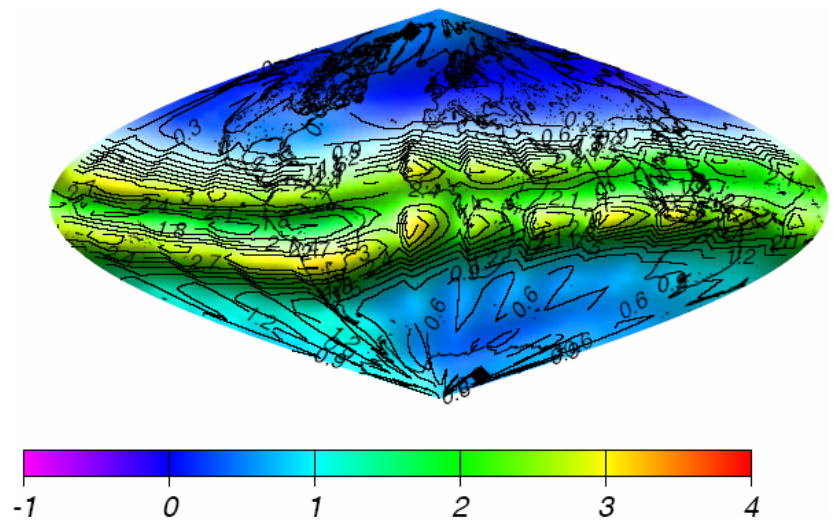

Fig. 5a. Spatial variation in the first mode for night conditions.

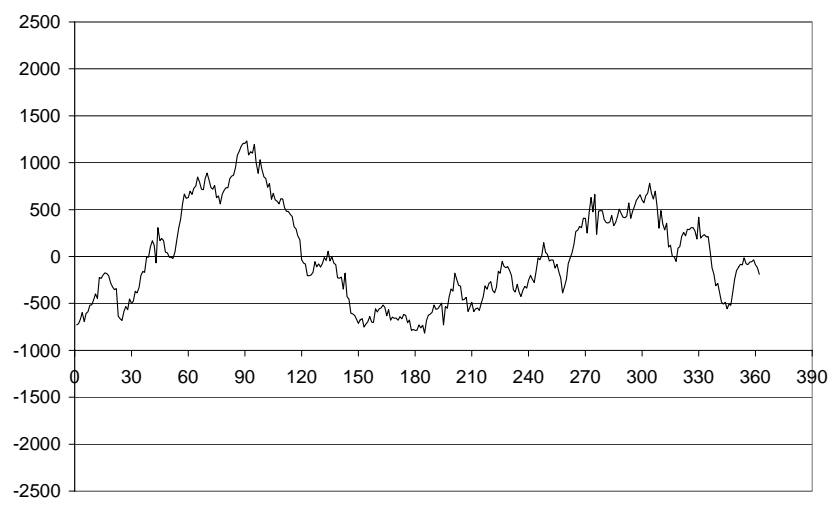

Fig. 5b. Time variation in the first mode for night conditions. The $\mathrm{x}$-axis is the day of year (DOY) and the $\mathrm{y}$-axis is the amplitude multiplied by a factor of $10^{-2}$.

the dataset. In the following we will analyze those factors instead of the VTEC variation itself. Figures 3 to 6 illustrate the spatial and temporal variation of VTEC for noon and night. Panels (a) represents the spatial variation $\left(e_{j}\right)$ and panels (b) the temporal variation $\left(a_{j}\right)$.

In all figures the geomagnetic poles are represented by diamonds. For Figs. $3 b$ to $6 b$ the $x$-axis represents the Day of Year (DOY) and the y-axis the amplitude. Equidistants for eigenvector contours in Fig. 3a is 0.2, Fig. 4a is 0.5 and Figs. $5 \mathrm{a}$ and $6 \mathrm{a}$ is 0.3 .

In the following we analyze the spatial and temporal variability factors for the first two modes and for different local time.

\subsection{Local time: noon}

The spatial variation, Fig. 3a, shows maximum values are distributed around the equatorial region and at low geomagnetic latitudes. As the geomagnetic latitude increases the VTEC variation decreases. The temporal variation, Fig. 3b, shows a semiannual variation with maximum in March-April and September-October. This figure shows the equinoctial 

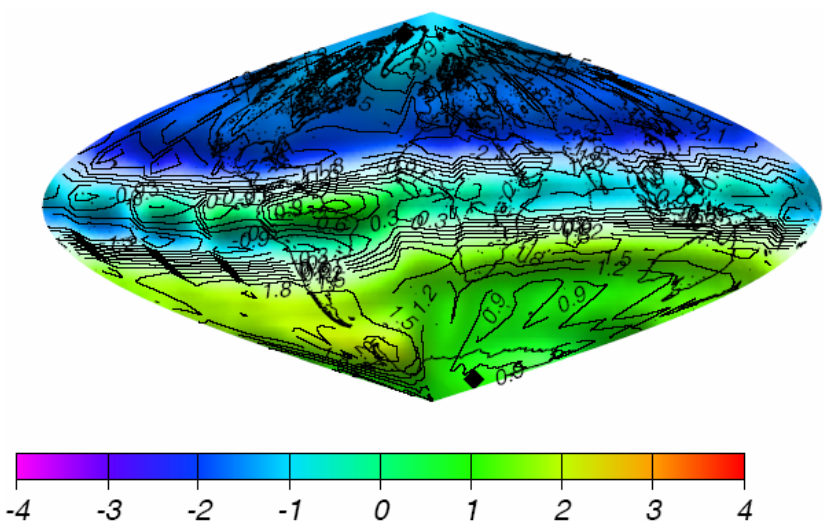

Fig. 6a. Spatial variation in the second mode at night.

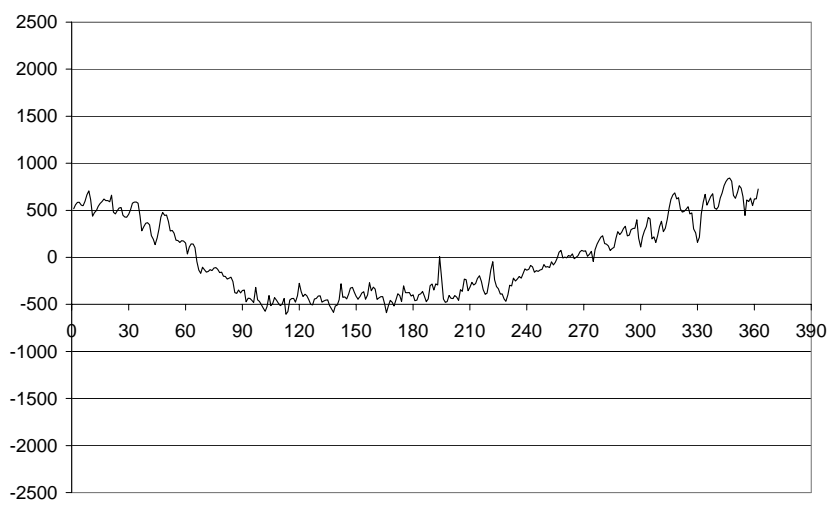

Fig. 6b. Time variation in the second mode at night. The $\mathrm{x}$-axis is the day of year (DOY) and the y-axis is the amplitude multiplied by a factor of $10^{-2}$.

asymmetry, being the March peak greater than September one.

The spatial variation, Fig. 4a, shows maximum positive values for low geomagnetic latitude in the Southern Hemisphere, principally in the South American region. In the north of Antarctica and between Africa and Oceania it is possible to see negative values and in the northern part of USA and Canada the spatial variation took positive values. For mid latitudes, in the Northern Hemisphere we found maximum negative values of spatial variation principally in the North African region and in the sector of Asia.

The temporal variation, Fig. 4b, shows an annual behavior with maximum values in December-January and minimum in June-July.

Using Eq. (7) for this mode, we found an annual variation for the entire globe, except in the north of Antarctica and between Africa and Oceania; and in Canada and the northern sector of USA.

\subsection{Local time: night}

The spatial variation, Fig. 5a, shows maximum values for low geomagnetic latitudes. As the geomagnetic latitude increases the variation decreases.

The temporal variation, Fig. 5b, shows a semiannual behavior with maximum values in March-April and September-October. It is possible to see the equinoctial asymmetry being March-April peak greater than SeptemberOctober one.

The spatial variation, Fig. 6a, shows maximum positive values in the Southern Hemisphere especially in the South American sector and maximum negative values in the Northern Hemisphere principally for mid geomagnetic latitudes and with a maximum positive value in South American sector.

The temporal variation, Fig. 6b, shows an annual variation with maximum values in December, especially in the northern sector of South America and minimum in June.

Analyzing both modes it is possible to see that the variability for night is lesser than the ones observed at noon.

Zhao et al. (2007) used global ionospheric maps of VTEC to study features of the annual and semiannual anomalies in the interval from 1999 to 2005 . These VTEC maps were, in turn, used to estimate the annual-to-mean amplitude ratio, $A_{1}$, and the semiannual-to-mean amplitude ratio, $A_{2}$, as well as the latitudinal symmetrical and asymmetrical parts, $A^{\prime}$ and $A^{\prime \prime}$ of A1, respectively. The author defines the symmetrical and asymmetrical indices as

$A^{\prime}=\left(A_{1}(\mathrm{MLAT})-A_{1}(-\mathrm{MLAT})\right) / 2$

$A^{\prime \prime}=\left(A_{1}(\mathrm{MLAT})+A_{1}(-\mathrm{MLAT})\right) / 2$

where

$A_{1}=\mathrm{TEC} 1 / \mathrm{TEC} 0 ; \quad A_{2}=\mathrm{TEC} 2 / \mathrm{TEC} 0$

TEC 1 is the annual and TEC2 the semiannual TEC components and TEC 0 is the annual average value of TEC and MLAT is the magnetic latitude.

$A^{\prime}$ denote the amplitude of the seasonal variation and $A^{\prime \prime}$ represents the annual anomaly.

Zhao et al. (2007) found that the symmetrical index $A^{\prime}$ manifests that noon winter anomaly has a solar activity dependence and during low solar activity the noon anomaly at mid latitudes is absent, with $A^{\prime \prime}$ being negative. The annual anomaly, represented by $A^{\prime \prime}$, showed to be more evident during solar activity such as 2002, except for year 2000, and least evident during low solar activity. The semiannual anomaly represented by the $A_{2}$ index, increases significantly with solar activity. Zhao found a pronounced double peak at low latitudes in the post-sunset, where it even reaches $40 \%$ during solar activity while being absent during low solar activity.

In our analysis $A_{1}$ and $A_{2}$ are related to the average over longitudes for each magnetic latitude for eigenvector $\left(\boldsymbol{e}_{j}(x)\right)$ 
Local noon 12:00 p.m.

Symmetric profile

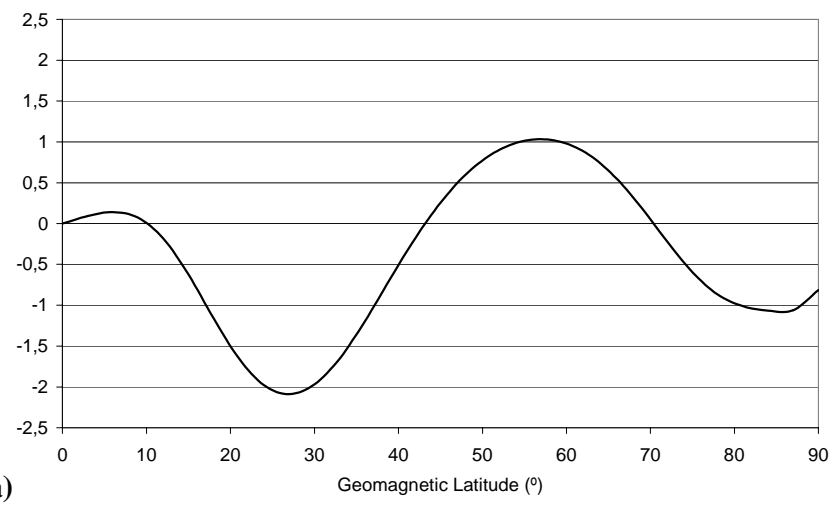

Asymmetric profile

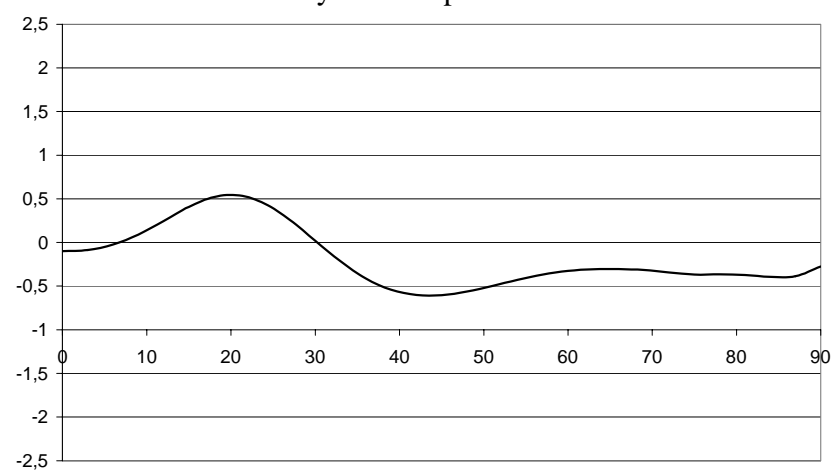

(b)

Geomagnetic Latitude $\left({ }^{\circ}\right)$

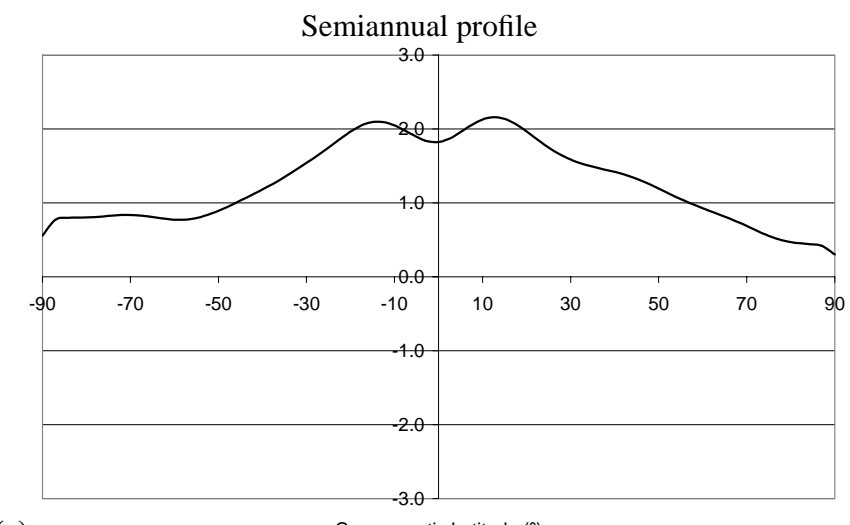

Local night 10:00 p.m.

Symmetric profile

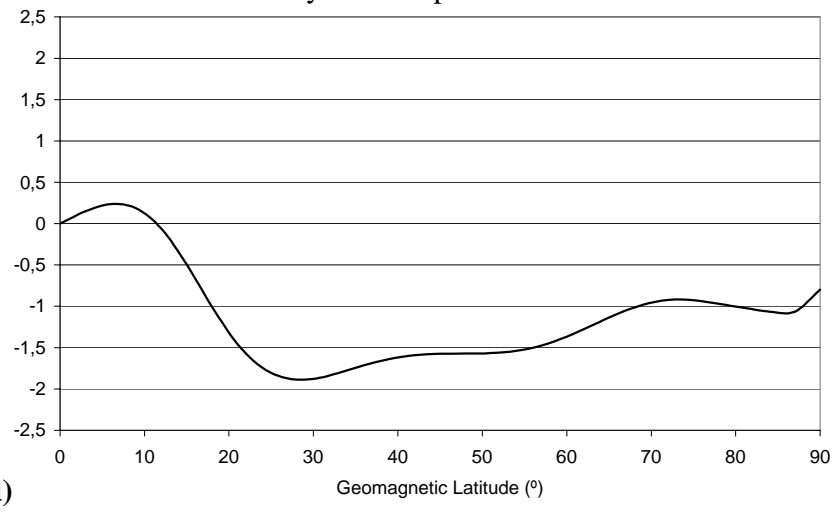

Asymmetric profile

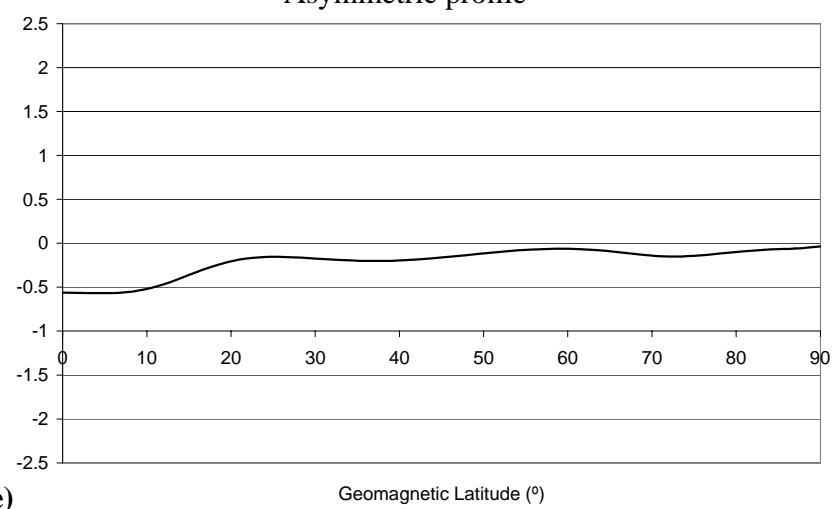

(e)

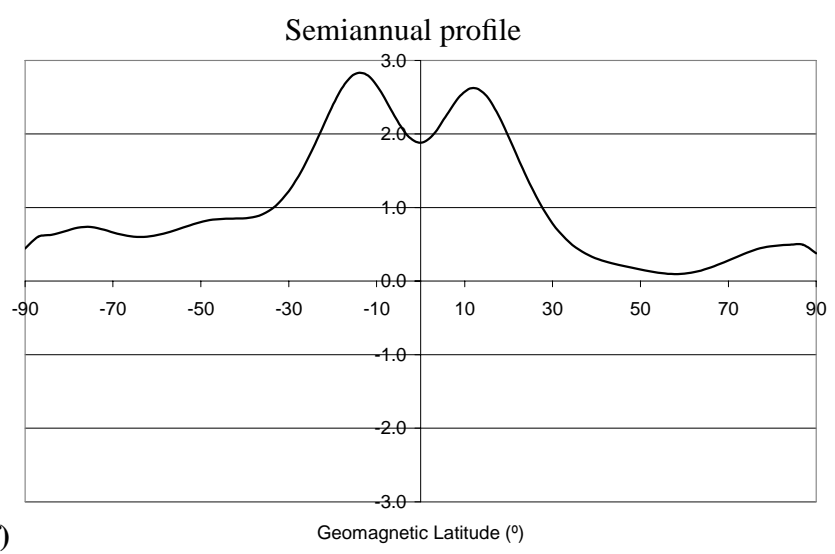

Fig. 7. Symmetric and asymmetric profile of $A_{1}$ and semiannual profile of $A_{2}$ for noon and night conditions.

of mode 2 and mode 1 , respectively. The study was made for noon and night. After that, Eqs. (8) and (9) were applied to this average, the result was the symmetric, asymmetric and semiannual profiles (Fig. 7a-f).

For local noon, Fig. 7a, shows a seasonal variation at high latitudes and Fig. 7b shows a weak annual anomaly at low latitudes, while in Fig. 7c is shown the semiannual anomaly at low latitudes with a slow decrease towards mid latitudes.
For local night, Fig. 7d and 7e show that there are neither seasonal nor annual anomalies. Figure $7 f$ shows a maximum semiannual variation at low latitudes with a sudden decrease towards mid latitudes.

It is possible to see a pronounced double peak structure for the night (Fig. 7f) which is not so evident during daytime (Fig. 7c). 


\section{Conclusions}

In this paper, we show once more the utility of the PCA technique to obtain features of the different anomalies. The application of PCA on VTEC data is a useful technique to analyze the spatial and temporal variability of the ionosphere. In this work it was possible to reconstruct $80 \%$ of the variability contained in global GPS derived VTEC data (for year 2000) using only the first two modes.

Both, the spatial distribution and the temporal variation are discussed for the first two modes. The main results are:

- PCA technique combined with IGS VTEC global maps offers time and spatial representation of the ionospheric variation.

- PCA is based on orthogonal decomposition of the data itself without forcing an annual and a semiannual component.

- For noon:

The first and second modes have significant contributions to the VTEC variance. The first mode represents the semiannual variation, with peaks on the equinoxes, being the March-April equinox higher than the September-October one with maximum values in the equatorial region and low geomagnetic latitudes. The variability of VTEC in March-April equinox exceeds the one of September-October equinox by up $40 \%$. The second mode shows the annual variation. We found seasonal anomaly at high latitude, a weak annual anomaly at low latitude and the semiannual anomaly at low latitude; those results are similar to the obtained by Zhao et al. (2007).

- For night:

The variations are smaller than those obtained at noon. The first mode shows a semiannual component with peaks on the equinoxes similar to the noon case, but with maximum values at low latitudes especially in the South American sector. The variability of VTEC in March-April equinox exceeds the variability during September-October equinox by up $50 \%$. The second mode shows an annual variation.

Comparing the results obtained for low solar activity using the same kind of data and numerical technique (Natali and Meza, 2010) we could conclude that the main characteristics between low and high solar activity are:

- We found a combination of semiannual and annual variation for low solar activity and a clear semiannual variation with peaks at the equinoxes for high solar activity.

- At night we found an annual component for low solar activity and a semiannual component for high solar activity.
- It is possible to see strong and weak annual variations for low latitudes at 12:00 p.m. and 10:00 p.m., respectively, for low solar activity without any seasonal anomaly while for high solar activity the seasonal anomaly is found at high latitudes at noon.

- The amplitude of semiannual variation is three times larger at high solar activity than at low solar activity and in both cases it is possible to see an asymmetrical structure between the two hemisphere.

- The annual anomaly is smaller by nighttime than noontime and decreases with increasing latitude. The amplitude of the annual anomaly is smaller than those obtained for low solar activity during daytime.

The IGS VTEC global maps produced by IGS have been demonstrating a great utility to do this analysis. The next step in this research is to analyze a complete solar cycle using the same numerical technique.

Acknowledgements. Topical Editor K. Kauristie thanks two anonymous referees for their help in evaluating this paper.

\section{References}

Astafyeva, E. I., Afraimovich, E. L., Oinats, A. V., Yasukevich, Y. V., and Zhivetiev, I. V.: Dynamics of global electron content in 1998-2005 derived from global GPS data and IRI modeling, Adv. Space Res., 42, 763-769, doi:10.1016/j.asr.2007.11.007, 2008.

Balan, N., Otsuka, Y., Bailey, G. J., Fukao, S., and Abdu, M. A.: Annual variations of the ionosphere: A review based on the MU radar observations, Adv. Space Res., 25, 153-162, 2000.

Buonsanto, M. J.: Possible effects of the changing Earth-Sun distance on the upper atmosphere, S. Pacific J. Nat. Sci., 8, 58-65, 1986.

Chaman Lal: Correlation between the seasonal trends of planetary F2 layer ion density and intensity of the ring current, J. Atmos. Solar-Terr. Phys., 57(1), 45-49, 1995.

Chaman Lal: Sun-Earth geometry, geomagnetic activity, and planetary F2 layer ion density, Part I: Signatures of magnetic reconnection, J. Atmos. Solar-Terr. Phys. 62, 3-16, 2000.

Chapman, S.: The absorption and dissociatival or ionizing effect of monochromatic radiation in atmosphere on a rotating earth, Proc. Phys. Soc., 43, 483-501, 1931.

Feltens, J. and Schaer, S.: IGS Products for the Ionosphere, in: Proceedings of the IGS Analysis Center Workshop, 225-232, edited by: Dow, J. M., Kouba, J., and Springer, T., Darmstadt, 9-11 February 1998.

Huang, Y. N. and Cheng, K.: Solar cycle variations of the equatorial ionospheric anomaly in total electron content in the Asian region, J. Geophys. Res., 101, 24513-24520, 1996.

Johnson, F. S.: Composition changes in the upper atmosphere, in: Electron Density Distributions in the Ionosphere and Exosphere, edited by: Thrane, E., North Holland, Amsterdam, 81-84, 1964.

$\mathrm{Li}, \mathrm{X}$. and $\mathrm{Yu}, \mathrm{T}$.: Annual and Semi-annual variations of the observed foF2 in a highsolar activity year, Terrestrial, Atmospheric and Oceanic Sciences, 14(1), 41-62, 2003. 
Liu, L., Wan, W., Ning, B., and Zhang, M.: Climatology of the mean total electron content derived from GPS global ionospheric maps, J. Geophys. Res., 114, A06308, doi:10.1029/2009JA014244, 2009.

Ma, R., Xu, J., and Liao, H.: The features and a possible mechanism of semiannual variation in the peak electron density of the low latitude F2 layer, J. Atmos. Solar-Terr. Phys., 65, 47-57, 2003.

Mayr, H. G. and Mahajan, K. K.: Seasonal variation in the F2 region, J. Geophys. Res., 76(4), 1017-1027, 1971.

Meza, A. and Natali, M. P.: Annual and semiannual TEC effects at low solar activity in midlatitude Atlantic region based on TOPEX, J. Geophys. Res., 113, D14115, doi:10.1029/2007JD009088, 2008.

Millward, G. H., Rishbeth, H., Fuller-Rowell, T. J., Aylward, A. D., Quegan, S., and Moffett, R. J.: Ionospheric F2 layer seasonal and semiannual variation, J. Geophys. Res., 101, 5149-5156, 1996.

Natali, M. P. and Meza, A.: Annual and semiannual VTEC effects at low solar activity based on GPS observations at different geomagnetic latitudes, J. Geophys. Res., 115, D18106, doi:10.1029/2010JD014267, 2010.

Nerem, R. S., Schrama, E. J., Koblinsky, C. J., and Beckley, B. D.: A preliminary evaluation of ocean topography from the TOPEX/POSEIDON mission, J. Geophys. Res., 99(C12), 24565-24583, 1994.

Nerem, R. S., Rachlin, K. E., and Beckley, B. D.: Characterization of global mean sea level variations observed by TOPEX/POSEIDON using empirical orthogonal functions, Surv. Geophys., 18, 293-302, 1997.

Numerical Recipes in FORTRAN 77: The Art of Scientific Computing (v. 1) by William H. Press, Brian P. Flannery, Cambridge University Press, 1995.

Preisendorfer, R. W.: Principal Component Analysis in Meteorology and Oceanography, 424 pp., Elsevier, Amsterdam, 1988.

Rishbeth, H.: How the thermospheric circulation affects the ionospheric F2-layer, J. Atmos. Solar-Terr. Phys., 60, 1385-1402, 1998.

Rishbeth, H. and Müller-Wodarg, I. C. F.: Why is there more ionosphere in January than in July? The annual asymmetry in the F2-layer, Ann. Geophys., 24, 3293-3311, doi:10.5194/angeo-243293-2006, 2006.

Rishbeth, H. and Setty, C. S. G. K.: The F-layer at sunrise, J. Atmos. Solar-Terr. Phys., 21, 263-276, 1961.

Rishbeth, H., Müller-Wodarg, I. C. F., Zou, L., Fuller-Rowell, T. J., Millward, G. H., Moffett, R. J., Idenden, D. W., and Aylward, A. D.: Annual and semiannual variations in the ionospheric F2-layer: II. Physical discussion, Ann. Geophys., 18, 945-956, doi:10.1007/s00585-000-0945-6, 2000.
Schaer, S., Beutler, G., and Rothacher, M.: Mapping and predicting the ionosphere, in: Proceedings of the IGS AC Workshop, Darmstadt, Germany, 1998.

Su, Y. Z., Bailey, G. J., and Oyama, K.-I.: Annual and seasonal variations in the low-latitude topside ionosphere, Ann. Geophys., 16, 974-985, doi:10.1007/s00585-998-0974-0, 1998.

Torr, M. R. and Torr, D. G.: The seasonal behavior of the F2-layer of the ionosphere, J. Atmos. Solar-Terr. Phys., 35(12), 2237-2251, 1973.

Volland, H.: The upper atmosphere as a multiple refractive medium for neutral air motions, J. Atmos. Solar-Terr. Phys., 31, 491-514, 1969.

Wu, C. C., Fryb, C. D., Liu, J. Y., Lioud, K., and Tseng, C. L.: Annual TEC variation in the equatorial anomaly region during the solar minimum: September 1996-August 1997, J. Atmos. Solar-Terr. Phys., 66, 199-207, 2004.

Yonezawa, T.: On the seasonal and non-seasonal annual variations and the semi-annual variation in the noon and midnight densities of the F2-layer in middle latitudes, II, J. Radio Res. Lab. Jpn., 6, 651-660, 1959.

Yonezawa, T.: The solar-activity and latitudinal characteristics of the seasonal, non-seasonal and semi-annual variations in the peak electron densities of the F2-layer at noon and at midnight in middle and low latitudes, J. Atmos. Solar-Terr. Phys., 33, 889-907, 1971.

Zhao, B., Wan, W., Liu, L., Yue, X., and Venkatraman, S.: Statistical characteristics of the total ion density in the topside ionosphere during the period 1996-2004 using empirical orthogonal function (EOF) analysis, Ann. Geophys., 23, 3615-3631, doi:10.5194/angeo-23-3615-2005, 2005.

Zhao, B., Wan, W., Liu, L., Mao, T., Ren, Z., Wang, M., and Christensen, A. B.: Features of annual and semiannual variations derived from the global ionospheric maps of total electron content, Ann. Geophys., 25, 2513-2527, doi:10.5194/angeo-252513-2007, 2007.

Zou, L., Rishbeth, H., Müller-Wodarg, I. C. F., Aylward, A. D., Millward, G. H., Fuller-Rowell, T. J., Idenden, D. W., and Moffett, R. J.: Annual and semiannual variations in the ionospheric F2-layer. I. Modelling, Ann. Geophys., 18, 927-944, doi:10.1007/s00585-000-0927-8, 2000. 